\title{
Free core nutation: direct observations and resonance effects
}

\author{
J. Vondrák ${ }^{1}$, R. Weber ${ }^{2}$, and C. Ron ${ }^{1}$ \\ 1 Astronomical Institute, Academy of Sciences of the Czech Republic, Boční II, 14131 Prague 4, Czech Republic \\ e-mail: vondrak; ron] @ig.cas.cz \\ 2 Institute of Geodesy and Geophysics, University of Technology Vienna, Gusshausstrasse 27-29, Vienna, 1040, Austria \\ e-mail: rweber@mars.hg.tuwien.ac .at
}

Received 13 May 2005 / Accepted 8 August 2005

\section{ABSTRACT}

The motion of Earth's spin axis in space is monitored by Very Long-Baseline Interferometry (VLBI), and since 1994 also its rate is measured by Global Positioning System (GPS). The method of "combined smoothing", developed recently at the Astronomical Institute in Prague enables one to combine both series. The solution, based on observations in 1979.6-2004.7, is compared with the recently adopted IAU2000 model of precession-nutation. The analysis reveals substantial residual offsets from the model. The dominant peak in frequency domain can be identified with Retrograde Free Core Nutation (RFCN), and we find also statistically significant deviations of several forced nutation terms. From the direct analysis of VLBI observations of celestial pole offsets from IAU2000 model of precession-nutation, it follows that the dominant period apparently grew from the average 435 days (VLBI observations in 1983.7-2004.7) to 460 days (VLBI/GPS combination in 1994.3-2004.6). A study of indirect determination of the RFCN period from the observed forced nutation terms through the resonance effects is presented, and the difference from the value found by direct analysis is discussed. It is demonstrated that the resonance approach does not confirm the apparent change of the period obtained from the direct analysis of observed celestial pole offsets; the resonance value is close to 430 solar days, and it seems to be very stable in time. The different value found from the direct analysis of observations can be probably ascribed to a small additional excitation by the atmosphere and/or ocean with retrograde terrestrial period around $23 \mathrm{~h} 53 \mathrm{~min}$ mean solar time.

Key words. astrometry - reference systems - Earth - techniques: miscellaneous - methods: numerical

\section{Introduction}

Precession-nutation, that describes variable orientation of the Earth's spin axis in space, has been observed since 1979 with a very high and ever increasing precision (nowadays of about 0.2 mas or better) by Very Long-Baseline Interferometry (VLBI). Thanks to these observations, a new model of precession-nutation IAU2000 was adopted by the IAU in 2000 . The new model is based on the theory of rotation of the rigid Earth REN-2000 by Souchay et al. (1999), containing forced terms exerted by the Moon, Sun and planets, and a transfer function calculated for a non-rigid Earth model (Mathews et al. 2002 - further referred to as MHB; Buffet et al. 2002), expressing frequency-dependent modification of the amplitudes and phases of individual nutation terms due to non-rigidity of the Earth. However, the model of nutation does not contain free motions that cannot be reliably predicted since they depend on integration constants and unknown excitations. They correspond to four eigenperiods of the Earth model - Chandler Wobble CW (with terrestrial period of about 435 days), Retrograde Free Core Nutation RFCN (with celestial period of about 430 days), Prograde Free Core Nutation PFCN (with celestial period of about 1020 days) and Inner Core Wobble ICW (with terrestrial period of about
2400 days). When observed in celestial reference frame, RFCN is dominant.

The Earth model used consists of the visco-elastic mantle, the fluid outer and the solid inner core, and includes also inner core anelasticity, ocean tide effects, electromagnetic couplings of the mantle and the solid inner core with the fluid outer core, annual atmospheric tide and relativistic effects (geodesic precession and nutation). Seven basic parameters of the model are estimated by a fit to VLBI observations of precessionnutation during the past two decades or so (Herring et al. 2002). Extragalactic sources are used by these observations as reference points, and therefore the EOP derived from VLBI are directly referred to the International Celestial Reference Frame - ICRF (Ma et al. 1998; Feissel \& Mignard 1998).

On the other hand, satellite methods measure the orientation of the Earth with respect to satellite orbits, and therefore are not capable of measuring the Earth's orientation directly with respect to the ICRF. Orientation of satellite orbits in space is subject to many influences, both gravitational and non-gravitational, and some of the orbital elements are correlated with some EOP, therefore their stable tie to ICRF is very difficult to model. Thus the satellite methods can provide only the time derivatives of those EOP that are sensitive to ICRF. The Global Positioning System (GPS), using the observations 
of navigation satellites, demonstrated its capability of measuring the rates of precession-nutation angles (Rothacher et al. 1999). As shown by Weber (1996, 2001), GPS observations can provide a contribution to the nutation spectrum in the high frequency range (up to 14 days).

The model IAU2000 is very close to the real motion of Earth's spin axis in space, so the celestial pole offsets measured by VLBI (deviations of its observed position from the model) are very small, typically smaller than 1 mas. However, some periodic terms can still be detected in the residuals, partly coming from the still existing deviations of the adopted model from reality, partly because of the presence of free nutations that are unpredictable and thus not included in the model. They are due to the fact that the outer fluid core and inner solid core can rotate around the axes that are not coincident with the spin axis of the mantle. Dominant is the Retrograde Free Core Nutation (RFCN) whose period in space is, roughly speaking, inversely proportional to the ellipticity of the outer fluid core $e_{\mathrm{f}}$ and electromagnetic coupling between the mantle and the outer core. This is one of the eigenperiods of the rotating Earth, which dominantly influences the transfer function between the rigid and non-rigid nutation theory. If the core were in hydrostatic equilibrium, its period would be around 460 mean solar days this value was used in the previous IAU1980 model of nutation (Wahr 1981); the new model IAU2000 however uses a value $e_{\mathrm{f}}$ by about $4 \%$ larger, leading to a period of about 430 days.

We have recently used VLBI observations, in combination with GPS data, to obtain combined solutions of celestial pole offsets in different time scales - Vondrák et al. (2002a,b) in a shorter time interval 1997.0-2000.0, Vondrák et al. (2003) in the interval 1994.3-2002.0, and most recently Vondrák \& Ron (2005) in the interval 1994.3-2004.7. To obtain these solutions, we used the method of combined smoothing (Vondrák \& Čepek 2000) with slight modifications.

Our recent studies (Vondrák \& Weber 2004; Vondrák \& Ron 2005) based on these combinations led to the conclusion that the "direct" RFCN observations (i.e., coming from the direct analysis of celestial pole offsets from the IAU2000 model) demonstrate that the period apparently grew in time from original 430 days to 460 days (i.e., closer to hydrostatic equilibrium value of $e_{\mathrm{f}}$ ) during the last 10 years. This result is however in contradiction with other authors (Roosbeek et al. 1999 or Hinderer et al. 2000) who concluded that the RFCN period is stable within 3 days, and is close to 430 solar days. Their analysis was based on older VLBI observations (1984-1998), and was also supported by the analysis of tidal gravity data in 1988-1996, from which Florsch \& Hinderer (2000) found the period of about 428 days.

In the direct analysis we usually tacitly assume that all forced nutations near RFCN frequency are covered by the model, so that only free motion is present in celestial pole offsets. However, it is well possible that the celestial pole offsets measured by VLBI contain not only the RFCN but also some non-modelled forced nutations with close frequencies, caused by geophysical excitations. Below we shall concentrate on a more detailed study of this effect; namely the indirect determination of RFCN period from resonance effects will be addressed, and compared with the direct determination.

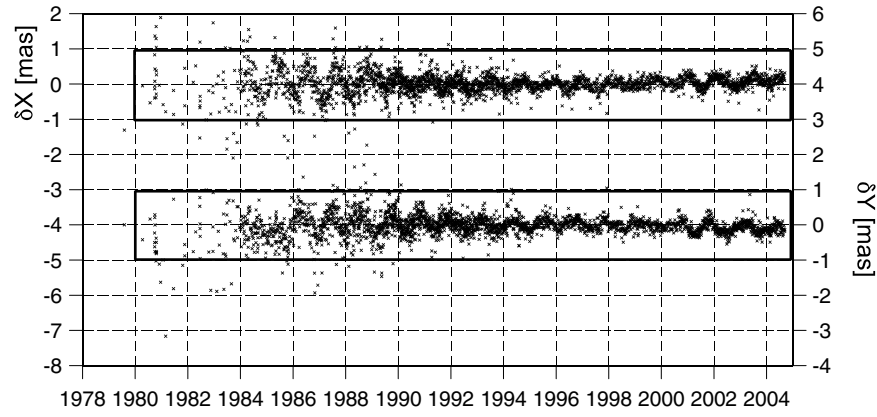

Fig. 1. Celestial pole offsets as measured by VLBI. The crosses mark individual observations, only the data inside the full-line boxes are used in subsequent analysis.

\section{The analysis of VLBI-only results}

Let us first have a look at VLBI data alone; the combined International VLBI Service (IVS) solution ivs $04 \mathrm{q} 3 \mathrm{X}$. eops is used in this study (Steinforth \& Nothnagel 2002; IVS 2005). The celestial pole offsets $\delta X, \delta Y$ with respect to the IAU2000 model of precession-nutation are displayed in Fig. 1. They express two components of the offset of the observed Celestial Intermediate Pole (CIP) from its position given by the adopted model. The relation between $\delta X, \delta Y$ and "classical" offsets in longitude $\delta \psi$ and obliquity $\delta \epsilon$, referred to the same model, can be approximated by simple relations

$$
\begin{aligned}
& \delta X=0.39778 \delta \psi+(0.02236 \delta \epsilon-0.00021 \delta \psi) T \\
& \delta Y=\delta \epsilon-0.00889 \delta \psi T,
\end{aligned}
$$

in which $T$ counts in centuries since $\mathbf{J} 2000.0$. The relations (1) are precise at least to $1 \mu$ as in the interval 1900-2100.

It is clear from Fig. 1 that the observations were much less precise at the beginning of the interval; we therefore "cleaned" the data by removing all of them whose absolute value exceeded 1 mas (see the corresponding boxes inside the figure). Almost all of the removed outliers were observed before 1990, but even the "cleaned" data in this period are still more noisy. It necessarily leads to somewhat worse determination of the amplitudes of nutation terms at the beginning of the interval studied.

Then the spectral analysis of the data were made (in complex form $\delta X+\mathrm{i} \delta Y$, in order to be able to distinguish prograde and retrograde motions), using Fast Fourier Transform (FFT) procedure with Parzen window (Press et al. 1992). Since equidistant arguments are needed for FFT, the data were interpolated for 3-day intervals. To get the interpolated data, very weak smoothing (with the coefficient of smoothing $\varepsilon=1$ ) was used (Vondrák 1969), and only then the linear interpolation was applied. Sparse data at the very beginning make the interpolation unreliable, so only the interval 1983.7-2004.7 is used. Spectral analysis was made for three different intervals, each 7 years long. The results are displayed in Fig. 2, where frequency is given in cycles per year [cpy]. The three spectra show that the amplitude of the dominant term gradually diminished, and its period grew - for the first interval (full line) the period is about 425 days, for the second one (dashed line) 430 days, and for the third one (dotted line) it is about 470 days. All these 


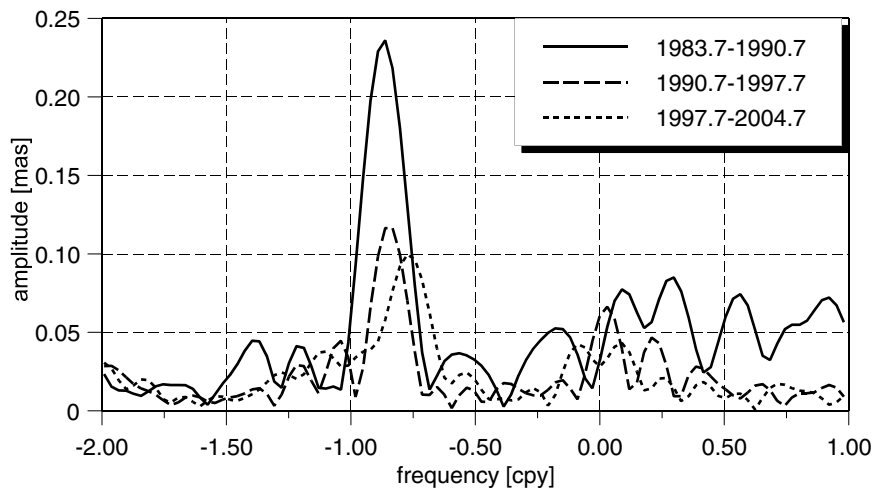

Fig. 2. FFT spectrum of VLBI celestial pole offsets in three different 7 -year intervals.

Table 1. Least-squares fit to IVS celestial pole offsets (bias, trend, RFCN and 3 long-periodic nutation terms, and their rms errors) in the interval 1980.0-2004.7.

\begin{tabular}{crrrrr}
\hline \hline Term & Period[d] & \multicolumn{2}{c}{$\delta X[\mu$ as $(/ \mathrm{cy})]$} & \multicolumn{2}{c}{$\delta Y[\mu \mathrm{as}(/ \mathrm{cy})]$} \\
& & $\cos$ & $\sin$ & $\cos$ & $\sin$ \\
\hline secular & $\infty$ & -6.0 & 15.2 & -38.6 & -19.7 \\
& & \pm 4.4 & \pm 4.2 & \pm 5.0 & \pm 4.7 \\
$\mathrm{RFCN}$ & -435 & -43.1 & -55.5 & -63.2 & 74.3 \\
& & \pm 4.3 & \pm 4.2 & \pm 4.8 & \pm 4.8 \\
$\Omega$ & -6798 & 39.5 & 11.6 & -58.1 & -1.8 \\
& & \pm 5.6 & \pm 7.4 & \pm 6.1 & \pm 8.0 \\
$2 \Omega$ & -3399 & 14.2 & -6.0 & -15.2 & -38.6 \\
& & \pm 5.3 & \pm 4.4 & \pm 5.7 & \pm 5.0 \\
$l^{\prime}$ & \multirow{2}{*}{365.26} & 15.2 & -15.0 & -19.7 & -37.4 \\
& & \pm 4.4 & \pm 4.3 & \pm 4.7 & \pm 4.9 \\
\hline
\end{tabular}

values are determined with rms errors of about 3 days, so their differences (exceeding the inaccuracies by an order of magnitude) are statistically significant. Average RFCN period for the whole interval is around 435 days. Recently Malkin (2004) also analyzed VLBI results, and came to similar conclusions; he demonstrated that the amplitude of RFCN was more or less steadily decreasing, and its period increased during the past years.

We used namely this average value of RFCN period to fit (using weighted least-squares method) cosine/sine terms of RFCN plus a bias, trend and corrections of three longperiodic nutation terms (with periods 18.6, 9.3 and 1 year), to the IVS data in the interval 1980.0-2004.7. The results are depicted in Table 1, in which the arguments $\Omega, l^{\prime}$ denote the mean longitude of the lunar ascending node and the mean anomaly of the Sun, respectively. It is evident that the deviations from the model are statistically significant (the corrections of the amplitudes exceed their rms errors by an order of magnitude).

Because of the ever changing amplitude and phase of RCFN, a simple sinusoidal model with a constant amplitude and period of 435 days is not fully adequate; in order to represent the free motion more realistically, we subtracted the bias, trend and the three nutation terms given in Table 1 from the data, and smoothed the residuals. 3D representation of the result is shown in Fig. 3, in which the time runs upwards along

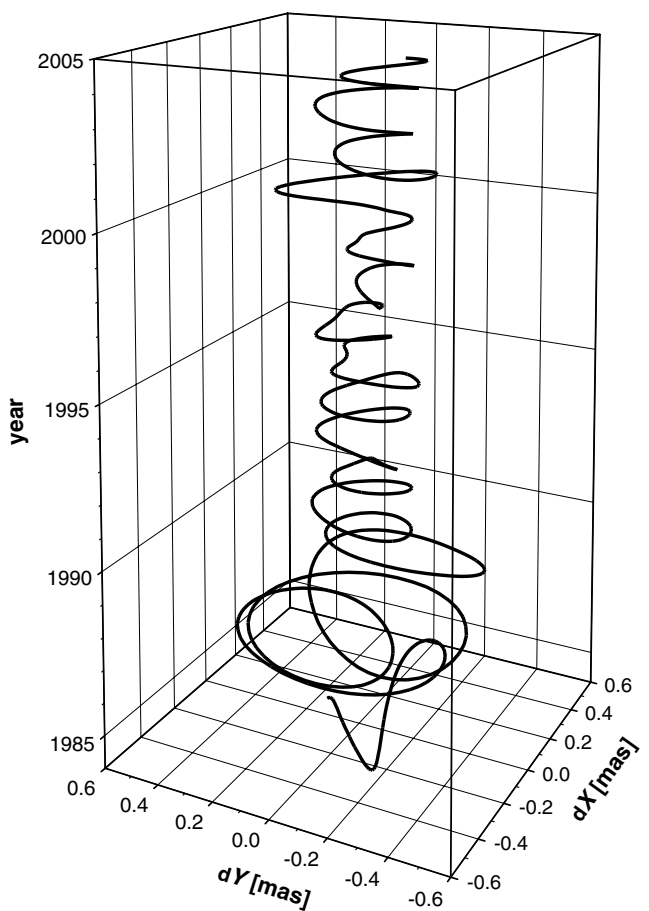

Fig. 3. Free core nutation from IVS observations, 1984.0-2004.7.

the $z$-axis. It clearly demonstrates how irregular this motion is, both in amplitude and phase.

\section{Combination of VLBI with GPS observations}

Now the IVS observations of celestial pole offsets are combined with their rates observed by GPS. The data provided by Center for Orbit Determination in Europe (CODE) for the interval 1994.3-2004.6 are used. Because they are still referred to the older nutation model IAU1980, IVS data $\delta \psi, \delta \epsilon$ referred to the same model had to be used; they are also available (solution ivs04q3e.eops). There is a qualitative difference between the two series: VLBI data are measured at unequally spaced epochs, typically between 1 and 7 days, and are very stable in time, while GPS observations are given at regular 1-day intervals, with a lower long-term stability due to problems with orbit modelling on longer intervals of time.

The combination was made using the "combined smoothing" method (Vondrák \& Čepek 2000, 2001). Since the method was described in detail many times in the past, we only remind here its principal philosophy. Two independently measured series:

- values of celestial pole offsets measured by VLBI; and

- values of celestial pole offset rates measured by GPS

are combined to obtain the solution that is

1. sufficiently smooth;

2. its function values fit well to the first series; and

3. its time derivatives fit well to the second series. 
The smoothness $S$ of the solution is defined by an integrated square of its third-order derivative, both fits $F, \bar{F}$ are defined in a least-squares sense, and the factor

$Q=S+\varepsilon F+\bar{\varepsilon} \bar{F}$

is minimized; $\varepsilon, \bar{\varepsilon} \geq 0$, called coefficients of smoothing, define the relative importance of the three constraints $S, F, \bar{F}$, and are to be chosen by an analyst. The method takes over the longterm stability of the first series, and combines it with the shortperiodic signal of the second one.

Before combination, GPS data were "calibrated" to fit, in a long-periodic sense (for periods 30 days and longer) to VLBI celestial pole offsets. The aim of the calibration was to remove the long-periodic part of GPS-based celestial pole offset rates that disagrees with VLBI observations and, at the same time, to retain the short-periodic part that is better monitored by GPS. To this end, we used a simple method similar to the one used by the IGS to derive Universal time from GPS-based length-ofday changes (Mireault et al. 1999; Kouba et al. 2000). Namely we integrated, at intervals covering integer number of VLBI epochs ( $8-15$ days long), the celestial pole offset rates by GPS and compared the result with the difference of celestial pole offsets by VLBI at the beginning and end of the interval. The difference, divided by the length of the interval, was then removed from each GPS value within the interval.

The method of combined smoothing was then applied to the corrected series. To compute the combination, we used the coefficients of smoothing $\varepsilon=1 \mathrm{day}^{-6}, \bar{\varepsilon}=0.2 \mathrm{day}^{-4}$, ensuring a reasonable relative weighting of VLBI and GPS data, and smoothing out all periods shorter than 2 days completely (for details on how to choose these parameters see Vondrák et al. 2002b).

Because the combination produced offsets $\delta \psi, \delta \epsilon$ referred to older IAU1980 model, they had to be corrected for the difference between IAU2000A and IAU1980 nutation tables, calculated by Ron (2002). We also removed from $\delta \psi, \delta \epsilon$ the values $\mathrm{d} \psi, \mathrm{d} \epsilon$, representing the small biases of the CIP at J2000.0 (McCarthy \& Capitaine 2002) and the linear corrections to IAU1976 precession. They are calculated from the formulas

$$
\begin{aligned}
\mathrm{d} \psi & =41.774+2.9965(t-2000.0), \\
\mathrm{d} \epsilon & =6.819+0.2524(t-2000.0)[\mathrm{mas}],
\end{aligned}
$$

in which $t$ is the time in years. Only then the offsets were transformed to $\delta X, \delta Y$, using relations (1).

The combination is displayed in Fig. 4 (full line), in which it is shown together with VLBI observations (crosses, triangles). High frequency signal of the combined series is due to GPS contribution. The results of a spectral analysis of the combination are displayed in Fig. 5, in which the horizontal dashed line depicts the 95\% significance level. Several significant peaks can be detected that may be identified with some forced nutation terms, but the dominant one, with period of about 460 solar days, can be connected with RFCN.

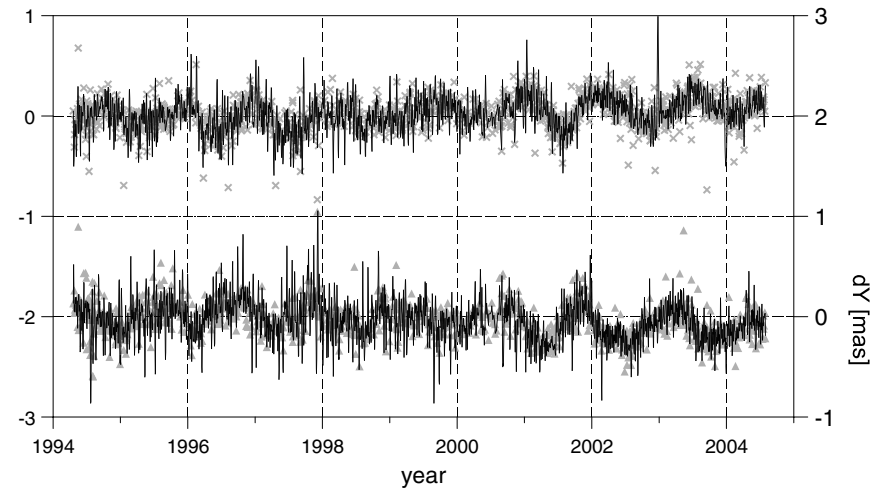

Fig. 4. Combined VLBI/GPS celestial pole offsets (full lines) and VLBI observations (crosses, triangles).
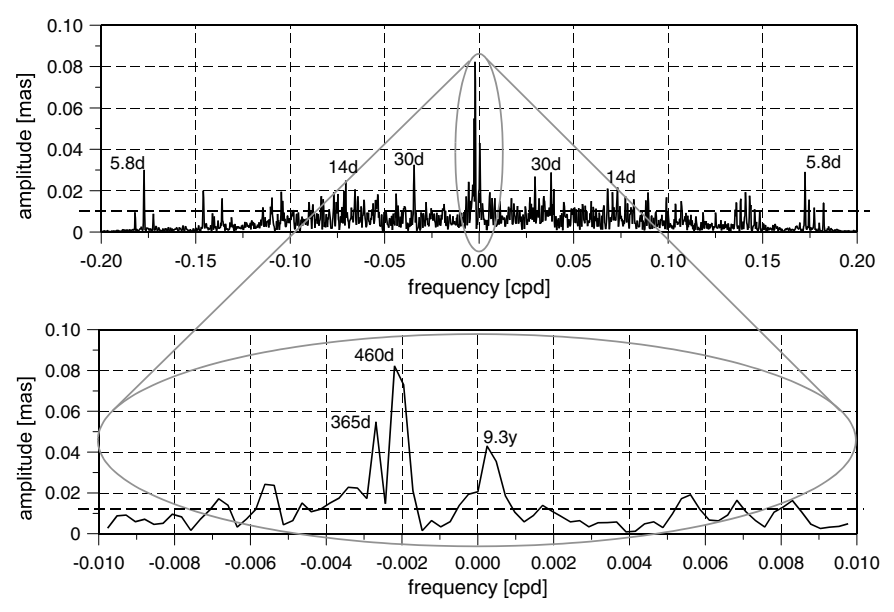

Fig. 5. Spectrum of combined VLBI/GPS celestial pole offsets in the interval 1994.3-2004.6; full spectrum (top) and close-up of its central part (bottom).

\section{Determination of RFCN period from resonance effects}

Now the question arises if the directly observed period of 460 days that we obtained from the analysis in the preceding section really implies the temporal change of the resonance period of RFCN. If it was the case, it should change the shape of the transfer function, and consequently also the amplitudes and phases of forced nutation terms (especially those with frequencies close to RFCN). We shall therefore first determine the amplitudes and phases of several dominant nutation terms from our VLBI/GPS combined solution for the last 10 years, and then use them, together with their theoretical rigid Earth values, to determine the RFCN resonance frequency.

The transfer function of IAU2000 model is given in MHB paper (Eq. (42)) as

$T(\sigma)=\frac{e_{\mathrm{R}}-\sigma}{e_{\mathrm{R}}+1} N_{\circ}\left[1+(1+\sigma)\left(Q_{\circ}+\sum_{j=1}^{4} \frac{Q_{j}}{\sigma-s_{j}}\right)\right]$

where $e_{\mathrm{R}}=(C-A) / A$ is the dynamical ellipticity of the rigid Earth used to compute the "rigid" solution; in our case, Souchay et al. (1999) used $H_{\mathrm{d}}=(C-A) / C=0.0032737548$ that yields $e_{\mathrm{R}}=H_{\mathrm{d}} /\left(1-H_{\mathrm{d}}\right)=0.0032845075$. The argument $\sigma$ 
Table 2. Parameters of MHB transfer function.

\begin{tabular}{rrr}
\hline \hline Parameter & $\mathrm{Re}$ & $\mathrm{Im}$ \\
\hline$N_{\circ}$ & 1.00001224 & - \\
$Q_{\circ}$ & -0.165291 & 0.0318995 \\
$Q_{1}$ & -0.948081 & 0.0678857 \\
$s_{1}$ & $3.11279 \times 10^{-3}$ & $3.76098 \times 10^{-4}$ \\
$Q_{2}$ & $4.89324 \times 10^{-2}$ & $1.61700 \times 10^{-3}$ \\
$s_{2}$ & -1.00231811 & $2.50607 \times 10^{-5}$ \\
$Q_{3}$ & $2.96114 \times 10^{-4}$ & $-9.56740 \times 10^{-5}$ \\
$s_{3}$ & -0.999026445 & $7.78663 \times 10^{-4}$ \\
$Q_{4}$ & $-1.10856 \times 10^{-5}$ & $-1.22654 \times 10^{-6}$ \\
$s_{4}$ & $4.1324 \times 10^{-4}$ & $9.28220 \times 10^{-8}$ \\
\hline
\end{tabular}

is the terrestrial frequency in cycles per sidereal day (cpsd), the other (generally complex) parameters of Eq. (4) are given in Table 2.

Parameters with subscripts 1 through 4 correspond to the four resonance modes of the wobbles $\mathrm{CW}, \mathrm{RFCN}, \mathrm{PFCN}$ and $\mathrm{ICW}$, respectively, their terrestrial frequencies $s_{1}-s_{4}$ are given in cpsd. Because the celestial and terrestrial frequency in cpsd are tied by a simple relation $\sigma_{\text {cel. }}=\sigma_{\text {ter. }}+1$, frequencies $s_{j}$ can be easily converted to corresponding periods (in mean solar days) in the terrestrial or celestial frame using the expressions

$$
\begin{aligned}
P_{j, \text { ter. }} & =0.99727 / s_{j} \\
P_{j, \text { cel. }} & =0.99727 /\left(s_{j}+1\right) .
\end{aligned}
$$

\subsection{Determination of "observed" values of transfer function for selected nutation terms}

To determine the "observed" values of the transfer function, we first removed the bias, trend and the two long-periodic terms (with arguments $\Omega, 2 \Omega$ ) found from the VLBI-only solution in Sect. 2 and shown in Table 1. Then we made a least-squares fit to determine cosine and sine terms in $\delta X, \delta Y$ of RFCN (with 460-day period) and five selected dominant forced nutations. The results are given in Table 3 , in which the arguments $l, F$ and $D$ denote the mean anomaly of the Moon, mean argument of its latitude and the mean elongation of the Moon from the Sun, respectively.

Each nutation term is generally an elliptic motion in celestial frame, and as such it can be expressed as a sum of two circular terms with different amplitudes and frequencies of opposite signs. If we denote cosine and sine corrections of a term in Table 3 in $\delta X, \delta Y$ as $C_{X}, S_{X}, C_{Y}, S_{Y}$ respectively, the corresponding corrections of prograde $\left(\Delta C^{+}\right)$and retrograde $\left(\Delta C^{-}\right)$ part of the same nutation term are given as

$\begin{aligned} \Delta C^{+} & =\frac{1}{2}\left[C_{X}+S_{Y}+\mathrm{i}\left(C_{Y}-S_{X}\right)\right] \\ \Delta C^{-} & =\frac{1}{2}\left[C_{X}-S_{Y}+\mathrm{i}\left(C_{Y}+S_{X}\right)\right] .\end{aligned}$

These corrections are then added to prograde and retrograde nutation terms as given by the model IAU2000A in order to obtain "observed" (complex) amplitudes of all five terms in question. Additional small corrections that are not due to the response of non-rigid Earth to luni-solar and planetary torques
Table 3. Least-squares fit to VLBI/GPS combined celestial pole offsets (RFCN and 5 dominant nutation terms, and their rms errors) in 1994.3-2004.6.

\begin{tabular}{crrrrr}
\hline \hline Term & Period [d] & \multicolumn{2}{c}{$\delta X[\mu$ as] } & \multicolumn{2}{c}{$\delta Y[\mu$ as] } \\
& & $\cos$ & $\sin$ & $\cos$ & sin \\
\hline RFCN & -460.00 & -21.8 & -88.0 & -95.6 & 38.6 \\
& & \pm 3.6 & \pm 3.6 & \pm 4.2 & \pm 4.3 \\
$l^{\prime}$ & 365.26 & 43.5 & -9.0 & 3.3 & -32.2 \\
& & \pm 3.6 & \pm 3.6 & \pm 4.2 & \pm 4.3 \\
$2 F-2 D+2 \Omega$ & 182.62 & -19.0 & 7.8 & -11.3 & 39.0 \\
& & \pm 3.6 & \pm 3.6 & \pm 4.2 & \pm 4.2 \\
$l^{\prime}+2 F-2 D+2 \Omega$ & 121.75 & -3.4 & -10.8 & 14.4 & 4.0 \\
& & \pm 3.6 & \pm 3.6 & \pm 4.2 & \pm 4.2 \\
$l$ & 27.55 & -1.2 & -5.1 & -10.8 & 6.6 \\
& & \pm 3.6 & \pm 3.6 & \pm 4.2 & \pm 4.2 \\
$2 F+2 \Omega$ & \multirow{2}{*}{25.66} & -17.9 & -17.6 & 13.8 & -10.0 \\
& & \pm 3.6 & \pm 3.6 & \pm 4.2 & \pm 4.2 \\
\hline
\end{tabular}

Table 4. Observed transfer function for 5 dominant nutation terms, based on combined VLBI/GPS solution in 1994.3-2004.6.

\begin{tabular}{rrrrr}
\hline \hline Period [d] & $\mathrm{Re} T^{+}$ & $\mathrm{Im} T^{+}$ & $\mathrm{Re} T^{-}$ & $\mathrm{Im} T^{-}$ \\
\hline 365.26 & 1.027043 & 0.001496 & 1.323730 & -0.008885 \\
& \pm 111 & \pm 111 & \pm 112 & \pm 112 \\
182.62 & 1.033343 & 0.001007 & 1.088239 & 0.002303 \\
& \pm 5 & \pm 5 & \pm 123 & \pm 123 \\
121.75 & 1.036402 & -0.000022 & 1.075171 & -0.0000109 \\
& \pm 134 & \pm 134 & \pm 3158 & \pm 3157 \\
27.55 & 1.037222 & -0.000145 & 1.062845 & 0.003521 \\
& \pm 198 & \pm 198 & \pm 213 & \pm 213 \\
13.66 & 1.029460 & -0.001600 & 1.072542 & 0.004108 \\
& \pm 30 & \pm 30 & \pm 814 & \pm 814 \\
\hline & & & &
\end{tabular}

(geodesic nutations, sun-synchronous corrections...), listed in Table 7 (Additions and Corrections...) of MHB, are then removed. The "observed" values of transfer function $T$ for these five terms are calculated, by dividing the "observed" amplitudes by their theoretical rigid-Earth values given by Souchay et al. (1999). Thus for each nutation term we obtain four values - real and imaginary part for both prograde and retrograde frequency. Table 4 shows the results, together with their mean errors that vary significantly for different nutation terms (and also for prograde and retrograde component), inversely depending on their rigid-body amplitudes.

These values are graphically depicted, together with the MHB transfer function calculated from Eq. (4), in Figs. 6 (real part) and 7 (imaginary part). The differences between the solid line (representing the MHB theoretical transfer function) and different symbols (representing the "observed" values for five largest nutation terms) are very small and hardly visible in this graphical representation.

\subsection{Determination of RFCN period}

Next we use the values of Table 4 to estimate the second resonance frequency $s_{2}$ of Eq. (4), corresponding to RFCN. To this end, we use the weighted least-squares method, in which the individual weights are inversely proportional to the squares 


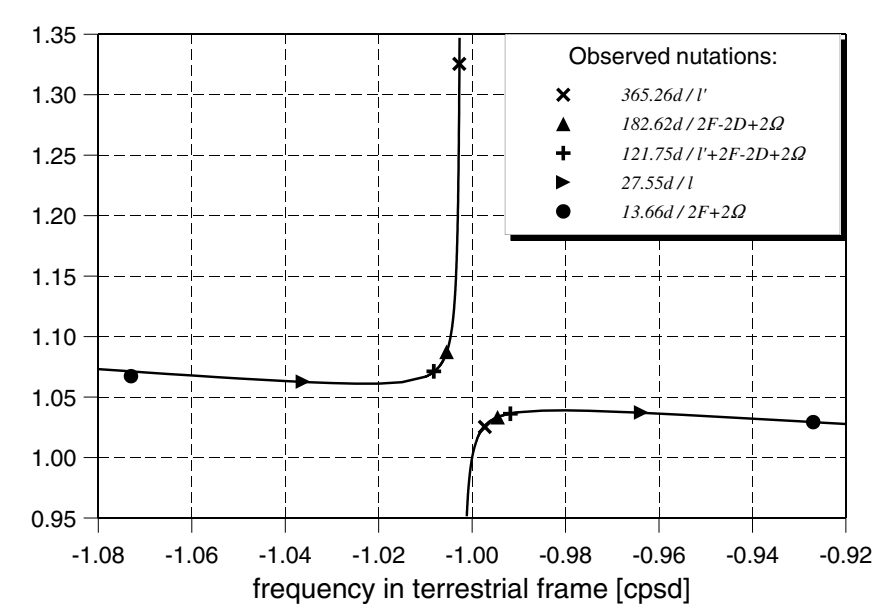

Fig. 6. Real part of MHB transfer function - comparison with the values for 5 nutation terms observed in 1994.3-2004.6.

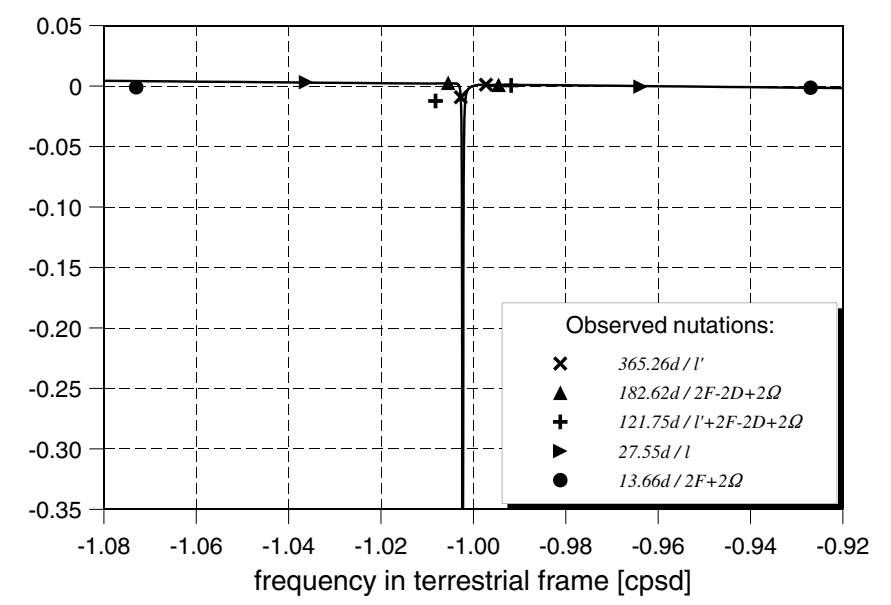

Fig. 7. Imaginary part of MHB transfer function - comparison with the values for 5 nutation terms observed in 1994.3-2004.6.

Table 5. RFCN frequency $s_{2}$ and celestial period estimated from resonance effects from the combined VLBI/GPS solution.

\begin{tabular}{crrr}
\hline \hline & $\operatorname{Re} s_{2}$ & $\operatorname{Im} s_{2}$ & $P_{\text {RFCN }}$ \\
\hline This study & -1.00231627 & 0.00002514 & -430.55 \\
& \pm 64 & \pm 63 & \pm 0.11 \\
MHB & -1.00231811 & 0.00002506 & -430.21 \\
& \pm 150 & \pm 150 & \pm 0.28 \\
\hline
\end{tabular}

of the mean errors. We leave all parameters of Eq. (4) intact, with the exception of two of them - real and imaginary part of $s_{2}$. To form the twenty observation equations (four per nutation term) we need the partial derivatives of transfer function with respect to $\operatorname{Re}\left(s_{2}\right), \operatorname{Im}\left(s_{2}\right)$. We calculate them numerically, by calculating $T$ for two close values of $s_{2}$ and dividing the difference of $T$ by the difference of the two values of $s_{2}$. The solution is then made by successive approximations. The result is shown in Table 5 and compared with the MHB value; periods are calculated by Eqs. (5).

It is clear that our and MHB values are almost identical, their difference is well below the standard errors. Our estimation, based on newer and more precise data, thus confirms the value used by MHB.

\section{Conclusions}

The apparent increase of RFCN period after 1994, detected by analysis of direct observations, is not confirmed by our analysis of resonance effects with the data covering the same interval. The new determination of the resonance period, based on largest five nutation terms (forced by luni-solar torques), derived from VLBI/GPS combined solution in 1994.68-2004.58 (with periods of $365.26,182.62,121.75,27.55$ and 13.66 days), is in good agreement with the value used by MHB. This period, equal to $430.55 \pm 0.11$ mean solar days, represents the proper resonance mode $s_{2}$ given by the properties of the internal structure of the Earth (mainly by the flattening of its fluid core).

On the other hand, the direct analysis of residual celestial pole offsets from MHB model leads to a different value of about 460 days. The difference can probably be explained by an additional excitation due to external parts of the Earth (atmospheric, oceanic, or a combination of both), as recently proposed, e.g., by Dehant et al. (2003). Its period in terrestrial frame can be found from Eqs. (5) as $P_{\text {ter. }}=P_{\text {cel. }} /\left(1-1.00274 P_{\text {cel. }}\right)$, provided the period in celestial frame is known. Putting $P_{\text {cel. }}=$ -460 days, we arrive at $P_{\text {ter. }}=-23 \mathrm{~h} 53 \mathrm{~min}$ mean solar time. Thanks to a close proximity of the resonance, any excitation with this period is extremely amplified so that the excitation necessary to explain the difference can be very small.

Acknowledgements. The Astronomical Institute is supported by Research Project No. AV0Z10030501. The authors also express their gratitude to the Grant Agency of the Academy of Sciences of the Czech Republic for the grant No. IAA3003205, to the Ministry of Education, Youth and Sports of the Czech Republic for supporting the Basic Research Center "Recent Dynamics of the Earth" LC506, IVS for providing the combined EOP solutions ivs $04 \mathrm{q} 3 \mathrm{X}$.eops, ivs04q3e.eops, and the Center for Orbit Determination in Europe (CODE) in Berne for providing the solution of celestial pole offset rates.

\section{References}

Buffet, B. A., Mathews, P. M., \& Herring, T. A. 2002, J. Geophys. Res., 107 (B4), 10.1029/2000JB000056

Dehant, V., Feissel-Vernier, M., de Viron, O., et al. 2003, J. Geophys. Res., 108 (B5), 10.1029/2002JB001763

Feissel, M., \& Mignard, F. 1998, A\&A, 331, L33

Florsch, N., \& Hinderer, J. 2000, Physics of the Earth and Planetary Interiors, 117, 21

Herring, T. A., Mathews, P. M., \& Buffet, B. A. 2002, J. Geophys. Res., 107 (B4), 10.1029/2001JB000165

Hinderer, J., Boy, J. P., Gegout, P., et al. 2000, Physics of the Earth and Planetary Interiors, 117, 37

IVS 2005, International VLBI Service products available at http://ivscc.gsfc. nasa.gov/service/products.html

Kouba, J., Beutler, G., \& Rothacher, M. 2000, in Polar Motion: Historical and Scientific problems, ed. S. Dick, D. McCarthy, \& B. Luzum, ASP Conf. Ser., 208, 277

Ma, C., Arias, E. F., Eubanks, M., et al. 1998, AJ, 116, 516

Malkin, Z. 2004, in Proc. IVS 2004 General Meeting, ed. N. R. Vandenberg, \& K. D. Baver (NASA/CP-2004-212255), 388

Mathews, P. M., Herring, T. A., \& Buffet, B. A. 2002, J. Geophys. Res., 107 (B4), 10.1029/2001JB000390 
McCarthy, D. D., \& Capitaine, N. 2002, in IERS Technical Notes 29, ed. N. Capitaine, et al. (BKG Frankfurt a.M.), 9

Mireault, Y., Kouba, J., \& Ray, J. 1999, GPS Solutions, Vol. 3, No. 1, 59

Press, W. H., Teukolsky, S. A., Vetterling, W. T., \& Flannery, B. P. 1992, Numerical Recipies in Fortran, 2nd ed. (Cambridge University Press) 490

Ron, C. 2002, in IERS Technical Notes 29, ed. N. Capitaine, et al. (BKG Frankfurt a.M.), 111

Roosbeek, F., Defraigne, P., Feissel, M., \& Dehant, V. 1999, Geophys. Res. Lett., 26, 131

Rothacher, M., Beutler, G., Herring, T. A., \& Weber, R. 1999, J. Geophys. Res., 104 (B3), 4835

Souchay, J., Loysel, B., Kinoshita, H., \& Folgueira, M. 1999, A\&A, 135,111

Steinforth, C., \& Nothnagel, N. 2002, in NASA/CP-2002-21002, IVS

General Meeting Proc., ed. N. R. Vanderberg, \& K. D. Baver, 265

Vondrák, J. 1969, Bull. Astron. Inst. Czechosl., 20, 349

Vondrák, J., \& Čepek, A. 2000, A\&AS, 147, 347
Vondrák, J., \& Čepek, A. 2001, in Journées 2000 Systèmes de référence spatio-temporels, ed. N. Capitaine (Observatoire de Paris), 252

Vondrák, J., \& Ron, C. 2005, Acta Geodynamica et Materialia, 2, No. 3 (139), 89

Vondrák, J., \& Weber, R. 2004, presented at AGU Fall Meeting, Session G03

Vondrák, J., Ron, C., Gambis, D., Bizouard, C., \& Weber, R. 2002a, in Journées 2001 Systèmes de référence spatio-temporels, ed. N. Capitaine (Observatoire de Paris \& Observatoire Royal de Belgique), 11

Vondrák, J., Weber, R., \& Ron, C. 2002b, in Vistas for Geodesy in the New Millennium, IAG Symp., 125, ed. J. Ádám, \& K. P. Schwartz (Berlin: Springer), 24

Vondrák, J., Ron, C., \& Weber, R. 2003, A\&A, 397, 771

Wahr, J. M. 1981, Geophys. J. Roy. Astron. Soc., 64, 651

Weber, R. 1996, ÖZf Vermessung \& Geoinformation, H3, 269

Weber, R. 2001, in Journées 2000 Systèmes de référence spatiotemporels, ed. N. Capitaine (Observatoire de Paris) 205 